\title{
Inactivation of Sirtuin2 protects mice from acetaminophen-induced liver injury: possible involvement of ER stress and S6K1 activation
}

\author{
Da Hyun Lee $e^{1,2, \#}$, Buhyun Lee $e^{1,2, \#}$, Jeong Su Park ${ }^{2}$, Yu Seol Lee ${ }^{1,2}$, Jin Hee Kim ${ }^{6}$, Yejin Cho ${ }^{2}$, Yoonjung Jo ${ }^{3}$, Hyun-Seok Kim ${ }^{3}$, \\ Yong-ho Lee ${ }^{4,5}$, , Ki Taek Nam ${ }^{1,2, *}$ E Soo Han Bae ${ }^{2, *}$ \\ ${ }^{1}$ Brain Korea 21 PLUS Project for Medical Science, Yonsei University, ${ }^{2}$ Severance Biomedical Science Institute, Yonsei Biomedical Research \\ Institute, Yonsei University College of Medicine, 50 Yonsei-ro, Seodaemun-gu, Seoul 03722, ${ }^{3}$ Department of Bioinspired Science, Ewha \\ Womans University, Seoul 120-750, ${ }^{4}$ Division of Endocrinology and Metabolism, Department of Internal Medicine, Yonsei University \\ College of Medicine, 50-1, Yonsei-ro, Seodaemun-gu, Seoul 03722, ${ }^{5}$ Institute of Endocrine Research, Yonsei University College of \\ Medicine, 50-1, Yonsei-ro, Seodaemun-gu, Seoul 03722, ${ }^{6}$ Brain Korea 21 PLUS Project for Medical Science, Yonsei University College of \\ Medicine, 50-1 Yonsei-ro, Seodaemun-gu, Seoul, 03722, Republic of Korea
}

\begin{abstract}
Acetaminophen (APAP) overdose can cause hepatotoxicity by inducing mitochondrial damage and subsequent necrosis in hepatocytes. Sirtuin2 (Sirt2) is an NAD ${ }^{+}$-dependent deacetylase that regulates several biological processes, including hepatic gluconeogenesis, as well as inflammatory pathways. We show that APAP decreases the expression of Sirt2. Moreover, the ablation of Sirt2 attenuates APAP-induced liver injuries, such as oxidative stress and mitochondrial damage in hepatocytes. We found that Sirt2 deficiency alleviates the APAP-mediated endoplasmic reticulum (ER) stress and phosphorylation of the p70 ribosomal S6 kinase 1 (S6K1). Moreover, Sirt2 interacts with and deacetylates S6K1, followed by S6K1 phosphorylation induction. This study elucidates the molecular mechanisms underlying the protective role of Sirt2 inactivation in APAP-induced liver injuries. [BMB Reports 2019; 52(3): 190-195]
\end{abstract}

\section{INTRODUCTION}

Drug-induced liver injury is a leading cause of acute liver failure. Therefore, the discovery of novel therapeutic targets for regulating the progression of liver injury is important (1).

*Corresponding authors. Soo Han Bae, Tel: +82-2-2228-0756; Fax: +82-2-2227-8129; E-mail: soohanbae@yuhs.ac; Ki Taek Nam, Tel: +82-2-2228-0754; Fax: +82-2-2227-8129; E-mail: KITAEK@yuhs.ac; Yong-ho Lee, Tel: +82-2-2228-1943; Fax: +82-2-393-6884; E-mail: yholee@yuhs.ac

${ }^{\#}$ These authors contributed equally to this work.

https://doi.org/10.5483/BMBRep.2019.52.3.083

Received 17 April 2018, Revised 10 May 2018, Accepted 13 July 2018

Keywords: Acetaminophen, ER stress, Hepatotoxicity, S6K1, Sirtuin2
Acetaminophen (APAP) has been widely used to understand the mechanism of drug-induced hepatotoxicity as a major cause of liver damage.

APAP-mediated hepatotoxicity is caused by the cytochrome P450 (CYP) 1A2-, CYP2E1-, and CYP3A4-driven conversion of APAP into hepatotoxic metabolites (2-4). Moreover, APAPinduced hepatotoxicity is associated with oxidative stress induced by $N$-Acetyl-p-benzoquinone imine, a reactive metabolite of APAP (5) or by endoplasmic reticulum (ER) stress $(6,7)$.

Sirtuins are $\mathrm{NAD}^{+}$-dependent deacetylases with seven isoforms (Sirt1-7) in mammals (8). Their functions are known to be associated with metabolic diseases, such as type II diabetes and obesity $(9,10)$. Among the seven isoforms, Sirtuin2 (Sirt2) may coordinate the regulation of several distinct metabolic processes, including adipocyte differentiation, fatty acid oxidation, hepatic gluconeogenesis, and insulin action, as well as the regulation of inflammatory pathways (11). Nevertheless, the role of Sirt2 in drug-induced liver injury remains unclear.

In this study, we investigated the role of Sirt2 in APAP-induced hepatotoxicity using mouse models in which Sirt2 was inactivated genetically and pharmacologically.

\section{RESULTS}

APAP decreases Sirt2 levels and Sirt2 ablation ameliorates APAP-induced liver injuries in mice

APAP is usually deemed as a safe drug, but APAP intoxication after an overdose can cause massive hepatocellular necrosis and acute liver injury $(2,4,5,12)$. To investigate whether Sirt2 plays a role in APAP-induced liver injuries, we first determined Sirt2 levels in the mouse liver following treatment with APAP for the indicated periods of time. Our results showed that APAP treatment induced the degradation of Sirt2 isoforms 1

ISSN: 1976-670X (electronic edition)

Copyright (c) 2019 by the The Korean Society for Biochemistry and Molecular Biology

(c) This is an open-access article distributed under the terms of the Creative Commons Attribution Non-Commercial License (http://creativecommons.org/licenses/by-nc/4.0) which permits unrestricted non-commercial use, distribution, and reproduction in any medium, provided the original work is properly cited. 

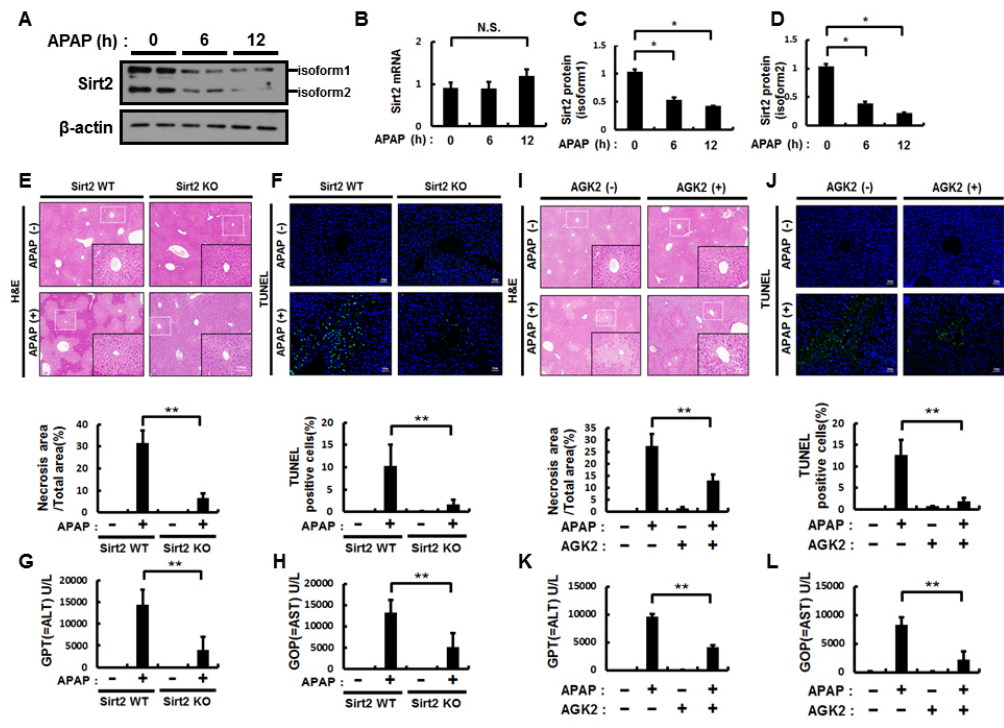

Fig. 1. APAP decreases Sirt2 levels in the mouse liver and ablation of Sirt2 ameliorates APAP-induced liver injuries in mice. (A) The livers of mice intraperitoneally injected with vehicle or APAP (500 mg/kg) for the indicated times were isolated, and liver homogenates were subjected to immunoblotting for Sirt2 and $\beta$-actin (loading control). (B) qRT-PCR analysis for the determination of Sirt2 mRNA levels. (C, D) Densitometric analysis of immunoblots, similar to those in (A). Ten-week-old WT and Sirt2 KO mice were injected with APAP (500 $\mathrm{mg} / \mathrm{kg}$ ). (E) Representative images, H\&E analysis (magnification, $100 \times$ ). The small panel images are enlarged photographs from the boxed areas (magnification, 200×), Quantitation of necrotic areas on H\&E-stained mouse liver sections. (F) TUNEL analysis of liver sections and quantitation of TUNEL analysis. (G) Serum levels of alanine transaminase (=GPT). (H) Serum levels of GOP. Investigation of the increased hepatotoxicity in mice treated with vehicle, APAP, or AGK2 (Sirt2 inhibitor). Ten-week-old WT and Sirt2 KO mice were injected with AGK2 (1 mg/kg) $2 \mathrm{~h}$ prior to APAP (500 mg/kg) administration, and plasma and livers were collected $12 \mathrm{~h}$ after APAP injection. (I) Representative images from H\&E analysis and Quantitation of the necrotic area. (J) TUNEL analysis and Quantitation. (K) Serum levels of alanine transaminase (=GPT). (L) Serum levels of GOP. Data represent the mean \pm SD from three independent experiments. $* P<0.05$, $* * \mathrm{P}<0.01, \mathrm{~N} . \mathrm{S}$, not significant.

and 2 in the mouse liver (Fig. 1A, 1C, 1D). However, the Sirt2 mRNA levels were not changed in the same samples (Fig. 1B). To verify whether Sirt2 degradation is regulated at the translational or post-transcriptional level, we treated normal liver cells (AML12 cells) with cycloheximide to inhibit protein synthesis and then measured Sirt2 levels in response to APAP-induced injuries. Sirt2 degradation was partially blocked in cycloheximide-treated AML12 cells (Supplementary Fig. 1A-1C), indicating that Sirt2 degradation is translationally modulated. We further examined whether downregulation of Sirt2 by APAP is mediated by the proteasomal or autophagic degradation pathway. AML12 cells were treated with an autophagy inhibitor (chloroquine, CQ) or a proteasome inhibitor (MG132). Immunoblot analysis showed that APAP-induced Sirt2 degradation was markedly inhibited by MG132 (Supplementary Fig. 1D-1F) and partially degraded by CQ (Supplementary Fig. 1G-1I). Taken together, these results indicate that APAP-induced downregulation of Sirt2 was regulated at the translational level and by the proteasomal degradation pathway. To investigate the effect of Sirt2 on APAP-induced liver injuries, we examined liver tissues from Sirt2 wild-type (WT) and Sirt2 knockout (KO) mice treated with APAP or vehicle for $12 \mathrm{~h}$. Furthermore, B6 mice, pre-treated with AGK2, the most potent Sirt2 inhibitor (13-15), were treated with APAP or vehicle to further examine whether the pharmacological inactivation of Sirt2 alleviates APAPinduced liver injuries. Subsequently, standard hematoxylineosin (H\&E) staining and TUNEL analysis were performed. Histological analyses using H\&E staining revealed that normal liver displayed prominent liver injuries and necrotic hepatic cell death. In contrast, histological sections from both Sirt2 KO mice (Fig. 1E) and mice treated with AGK2 (Fig. 1I) showed a decrease in the severity of liver injuries and necrotic hepatic cell death compared with Sirt2 WT mice in response to APAP. Moreover, TUNEL-positive apoptotic cells were scarcely detected in the livers of Sirt2 WT and Sirt2 KO mice treated with APAP or vehicle for $12 \mathrm{~h}$. Our results showed a considerable decrease in the percentage of TUNEL-positive cells in the liver of Sirt2 KO mice (Fig. 1F) and mice treated with AGK2 (Fig. 1J) in response to APAP. Moreover, Sirt2 KO mice treated with APAP and mice treated with AGK2 exhibited decreased levels of alanine transaminase (GPT) and aspartate transaminase (GOP) in response to APAP (Fig. 1G-1H, 1K-1L). Correspondingly, Sirt2 KO mice treated with APAP for $6 \mathrm{~h}$ 
showed a substantial decrease in the size of necrotic areas, TUNEL-positive cell count, and GPT and GOP levels (Supplementary Fig. 2A-2F). Furthermore, we found that the level of protein 3-nitrotyrosine, a marker of oxidative stress, decreased in Sirt2 KO mice treated with APAP for $12 \mathrm{~h}$ (Supplementary Fig. 3A, 3B) and 6 h (Supplementary Fig. 4A, 4B), and in AGK2-treated livers (Supplementary Fig. 5A, 5B), compared to that in Sirt2 WT mice. Based on the notion that an APAP overdose significantly induces mitochondrial damage (16), we provide evidence that the ablation of Sirt2 and pharmacological inactivation of Sirt2 alleviate the APAPinduced mitochondrial damage, using electron microscopy. In the same samples, we found distinctive mitochondrial morphological changes, typical necrotic cells with swollen mitochondria, accumulation of lipid droplets, and disruption of plasma membranes (17) (Supplementary Fig. 3C, 5C). Collectively, our data show that the pharmacological inactivation of Sirt2 in APAP-treated mouse livers exhibits effects similar to those of the pathology that results from the ablation of Sirt2.

The inactivation of Sirt2 attenuates ER stress in APAP-induced liver injuries in mice

It has been reported that APAP can induce ER stress in vivo and in vitro and that these deleterious effects can play a significant role in the APAP-induced cell death in the liver (6, 18-20). Upon acute ER stress, BiP/Grp78 dissociates from

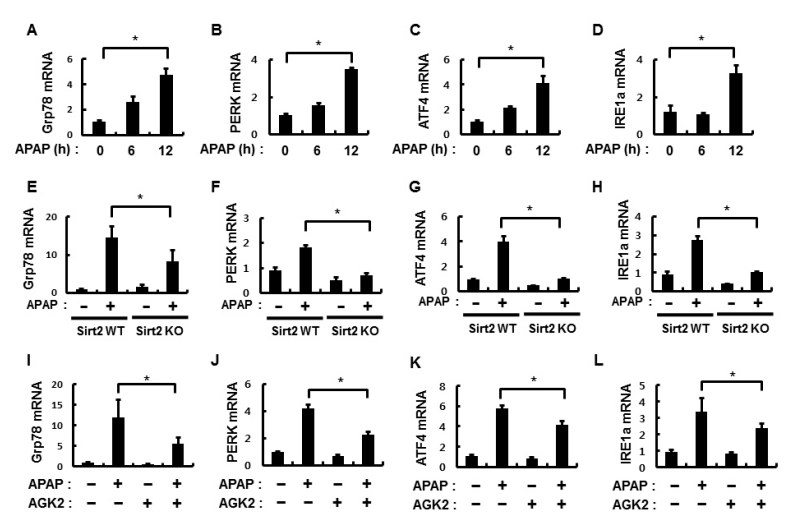

Fig. 2. The inactivation of Sirt2 attenuates ER stress in APAP-induced liver injuries in mice. (A-D) The livers of mice intraperitoneally injected with vehicle or APAP $(500 \mathrm{mg} / \mathrm{kg}$ ) for the indicated times were isolated, and GRT-PCR analysis for the determination of Grp78, PERK, ATF4, and IRE1 $\alpha$ mRNA levels. (E-H) Sirt2 WT or Sirt2 KO mice $12 \mathrm{~h}$ after an intraperitoneal injected with vehicle or APAP $(500 \mathrm{mg} / \mathrm{kg})$. qRT-PCR analysis for the determination of Grp78, PERK, ATF4, and IRE1 $\alpha$ mRNA levels. (I-L) The livers of mice intraperitoneally injected with vehicle, APAP (500 mg/kg), and AGK2 (1 mg/kg) for $12 \mathrm{~h}$ were isolated, and qRT-PCR analysis for the determination of Grp78, PERK, ATF4, and IRE $1 \alpha$ mRNA levels. Data represent the mean \pm SD from three independent experiments. ${ }^{*} \mathrm{P}<0.05$. these sensors, leading to the activation of the unfolded protein response pathway, to resolve ER stress and restore homeostasis (21). Consistent with this report, we found that ER stress marker genes such as BiP/Grp78, PERK, ATF4, and IRE1 $\alpha$ were also upregulated in the mouse liver (Fig. 2A-2D). To determine whether Sirt2 regulates the APAP-mediated increase in ER stress, we examined the levels of ER stress markers in Sirt2 WT and $\mathrm{KO}$ mice treated with APAP. APAP enhanced BiP/Grp78 levels and downregulated ER stress marker genes in Sirt2 KO mice (Fig. 2E-2H) and mice treated with AGK2 (Fig. 2I-2L), as assessed by qRT-PCR. Moreover, the short APAP treatment $(6$ h) showed a similar effect (Supplementary Fig. 6A-6E). Consistent with the notion that ablation of Sirt2 regulates the APAP-induced ER stress, these results suggest that inactivation of Sirt2 ameliorates the APAP-induced ER stress in the mouse liver.

\section{The APAP-induced S6K1 phosphorylation is inhibited in the livers of Sirt2-inactivated mice}

The mammalian target of rapamycin complex 1 (mTORC1) has been implicated in the regulation of ER stress, and the inhibition of mTORC1 may have potential therapeutic effects in ER stress-related diseases (22). To investigate whether the ablation of Sirt2 ameliorates the APAP-induced ER stress through the regulation of the mTORC1 signaling pathway, the level of mTOR phosphorylation induced by APAP was determined by immunoblot analysis in Sirt2 WT and KO mice.

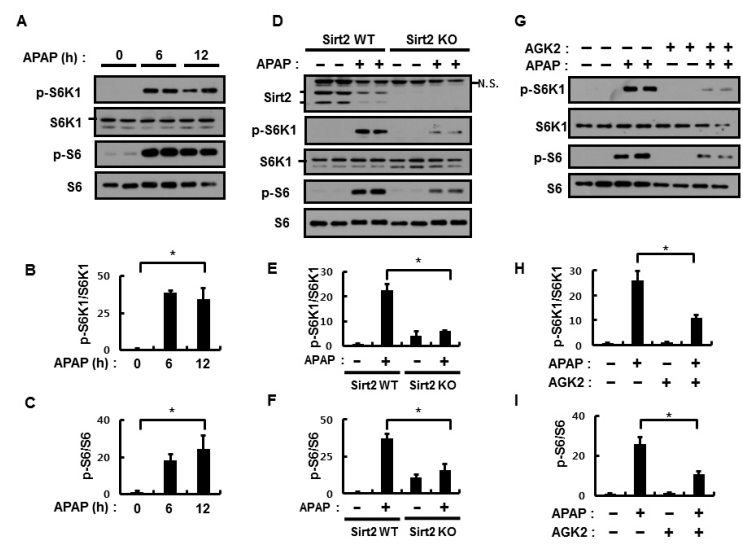

Fig. 3. The APAP-induced S6K1 phosphorylation is inhibited in the livers of Sirt2-inactivated mice. (A) The livers of mice intraperitoneally injected with vehicle or APAP $(500 \mathrm{mg} / \mathrm{kg})$ for the indicated times were isolated, and immunoblot analysis for p-S6K1, S6K1, p-S6, and S6. (B, C) Densitometric analysis. (D-F) Sirt2 WT or Sirt2 KO mice $12 \mathrm{~h}$ after an intraperitoneal injected with vehicle or APAP (500 mg/kg), immunoblot analysis for Sirt2, p-S6K1, S6K1, p-S6, and S6. Densitometric analysis (D). (G) The livers of mice intraperitoneally injected with vehicle, APAP (500 $\mathrm{mg} / \mathrm{kg}), \operatorname{AGK} 2(1 \mathrm{mg} / \mathrm{kg})$ for $12 \mathrm{~h}$ were isolated, and immunoblot analyses for p-S6K1, S6K1, p-S6, and S6. (H, I) Densitometric analysis. Data represent the mean \pm SD from three independent experiments. ${ }^{*} \mathrm{P}<0.05$. 
Our results indicated that mTOR phosphorylation level did not differ between the two groups (Supplementary Fig. 7A-7D). S6K1 and ribosomal protein S6 are downstream targets of mTORC1. Chronic acetaminophen treatment increased S6K1 phosphorylation in rat muscles (23). To investigate whether Sirt2 regulates the S6K1 signaling pathway, we examined the levels of APAP-induced S6K1 phosphorylation in the livers of Sirt2 WT and KO mice and mice treated with AGK2. In accordance with the results obtained in rats, we observed that the phosphorylation of S6K1 and that of the S6 ribosomal protein gradually increased in a time-dependent manner following APAP treatment in the mouse liver (Fig. 3A-3C). Moreover, S6K1 phosphorylation was markedly decreased in Sirt2 KO mice treated with APAP for $12 \mathrm{~h}$ (Fig. 3D-3F), $6 \mathrm{~h}$ (Supplementary Fig. 6A, 6B) and in mice treated with AGK2 (Fig. 3G-3I). These results confirmed that Sirt2 inactivation downregulates the APAP-induced ER stress by inhibiting the S6K1 signaling pathway.

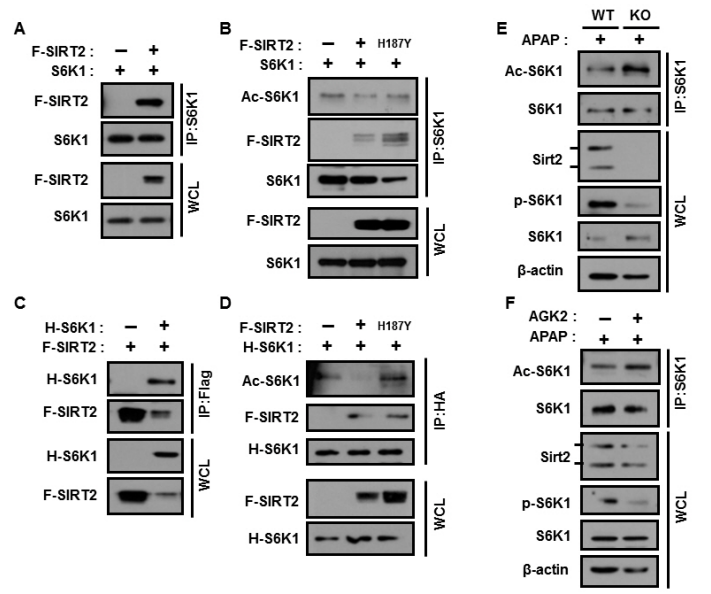

Fig. 4. Sirt2 regulates the phosphorylation of S6K1 through S6K1 deacetylation in APAP-treated mouse livers. Immunoblots of immunoprecipitates and whole-cell lysates from lysates of (A) HEK293 cells transfected with S6K1 and Flag-Sirt2 (F-SIRT2) vectors subjected to immunoprecipitation with an antibody against S6K1. (B) HEK293 cells transfected with an S6K1, F-SIRT2, and a Sirt2 (H187Y) catalytic dead mutant vector subjected to immunoprecipitation with antibodies against acetyllysine, S6K1, or Flag. (C) HEK293 cells transfected with an HA-S6K1 (H-S6K1) or a Flag-Sirt2 vector subjected to immunoprecipitation with antibodies against Flag. (D) HEK293 cells transfected with H-S6K1, F-SIRT2, and F-SIRT2 (H187Y) vectors subjected to immunoprecipitation with antibodies against acetyllysine, or HA. (E) Sirt2 WT or Sirt2 $\mathrm{KO}$ mouse livers, following treatment with APAP for $12 \mathrm{~h}$, subjected to immunoprecipitation with an anti-S6K1 antibody. (F) Sirt2 WT mouse livers, following treatment with APAP or AGK2 for $12 \mathrm{~h}$, subjected to immunoprecipitation with an with an antibody against S6K1.

\section{Sirt2 regulates S6K1 phosphorylation through S6K1 deacetylation in APAP-treated mouse livers}

It has been reported that S6K1 acetylation blocks the mTORC1-dependent S6K1 phosphorylation at T389, an essential phosphorylation site for S6K1 activity and that this acetylation is inhibited by Sirt2 (24). Therefore, we explored the mechanism underlying the Sirt2-mediated regulation of S6K1 acetylation in APAP-treated mouse livers. Firstly, to confirm the role of Sirt2 in S6K1 deacetylation, we performed a co-immunoprecipitation analysis on HEK293 cells transfected with expression vectors for S6K1 or HA-tagged S6K1, together with a vector encoding a Flag-tagged Sirt2 (F-SIRT2). Consistent with the above report, our results showed that Sirt2 interacts with S6K1 (Fig. 4A, 4C). Furthermore, to validate that S6K1 deacetylation is mediated by Sirt2, we performed an acetylation assay coupled with co-immunoprecipitation in HEK293 cells transfected with expression vectors for the wild-type or a Sirt2 catalytic mutant $(\mathrm{H} 187 \mathrm{Y})$ and S6K1 or HA-tagged S6K1. Our results revealed that the Sirt2 catalytic mutant did not deacetylate S6K1, as opposed to the wild-type Sirt2 (Fig. 4B, 4D). In addition, we observed similar results using APAP-treated livers from Sirt2 knockout mice and AGK2-pretreated mice (Fig. 4E, 4F). Taken together, these results suggest that Sirt2 interacts with and deacetylates S6K1 to promotes its phosphorylation.

\section{DISCUSSION}

In this study, we found that an APAP overdose decreased the expression of two Sirt2 proteins in the mouse liver. A sequence analysis, using the GenBank sequence database, showed that Sirt2 has four different human splice variants. However, only transcript variants 1 and 2 have been verified as protein isoforms of physiological relevance (25).

The observation that APAP may mediate the downregulation of Sirt2 proteins (isoforms 1 and 2) led us to investigate the role of Sirt2 in APAP-induced liver injuries. Our results revealed that the Sirt2 deficiency renders mice less susceptible to APAP-induced hepatotoxicity.

Recent screening campaigns have identified several selective inhibitors of Sirt2, including Sirt-rearranging ligands 1 and 2, AK-1, AK-7, and AGK2 $(26,27)$. Of these, AGK2 is the most potent inhibitor of Sirt2 $(13,15)$. Accordingly, we found that AGK2 treatment significantly alleviated the APAP-induced hepatotoxicity in the mouse liver.

Excessive ER stress results in multiple pathologies, including cirrhosis, non-alcoholic fatty liver disease, diabetes mellitus, viral inflammation, and cancer $(28,29)$. Previous studies have reported that APAP is able to induce ER stress in vivo and in vitro and that this deleterious effect could play a significant role in the APAP-induced cell death in the liver $(6,18-20)$. Our observation that the Sirt2 deficiency attenuates the APAPinduced ER stress might explain the protective role of Sirt2 inactivation in the APAP-induced hepatotoxicity. 
It is widely known that mTORC1 regulates cellular processes, protein synthesis, cell growth, and survival through the phosphorylation and activation of S6K1 (30). Moreover, mTORC1 represents one of the upstream triggers of ER stress; hence. the inhibition of mTORC1 may potentially be employed in the therapy of ER stress-induced diseases (22). A previous study reported that $\mathrm{mTOR} / \mathrm{S} 6 \mathrm{~K}$ signaling is inhibited by acetaminophen; however, this study was limited as primary mouse hepatocytes were used for the experiments $(17,31)$. On the contrary, there are several reports that S6K1 phosphorylation is increased by chronic APAP treatment in rats (23) and by APAP overdose in mouse livers $(32,33)$. Correspondingly, our results showed that the ablation of Sirt2 downregulates the APAP-induced S6K1 phosphorylation, resulting in an attenuation of ER stress. However, the inactivation of Sirt2 has no effect on the phosphorylation of mTORC1 (Supplementary Fig. 7). These observations suggest that S6K1 may be a specific substrate of Sirt2 in this context.

A recent study has shown that the acetylation of S6K1 blocks the mTORC1-dependent phosphorylation of S6K1 and that the acetylation of S6K1 is inhibited by Sirt2 (24). In agreement with this report, we also found that Sirt2 interacts with and deacetylates S6K1. Thus, Sirt2 inhibition induces S6K1 acetylation and subsequently block S6K1 phosphorylation to downregulate the APAP-induced ER stress in the mouse liver.

Growing evidence from recent studies indicates that the inactivation of Sirt2 has protective roles in liver diseases. For example, it has been reported that Sirt2 deficiency might attenuate the APAP-induced liver toxicity by downregulating c-Jun $\mathrm{NH} 2$-terminal kinase (JNK) activation. Furthermore, Sirt2 aggravates the hepatic ischemia-reperfusion injury through the deacetylation and inhibition of mitogen-activated protein kinase phosphatase-1 (34). Moreover, Sirt2 accelerates hepatic fibrogenesis by regulating the ERK/c-MYC pathway. Therefore, the inactivation of Sirt2 may represent an effective strategy for protecting against the development of hepatic fibrosis (35). In addition, the ablation of the Sirt3 mitochondrial deacetylase plays a protective role in APAP-induced hepatotoxicity through the activation of mitochondrial aldehyde dehydrogenase 2 (36).

Furthermore, Sirt2 knockdown induces autophagy and prevents post-slippage death by abnormally prolonging the mitotic arrest induced by microtubule inhibitors (37). Moreover, Sirt2 leads to genetic instability and tumorigenesis by positively regulating APC/C activity via the deacetylation of its coactivators, $\mathrm{CDH} 1$ and $\mathrm{CDC} 20(38,39)$.

The silencing of Sirt2 significantly activates necrosis in PC12 cells but does not affect autophagy in these cells (40). Furthermore, the inhibition of Sirt2 induces p53-dependent apoptosis in cancer cells (41). In conclusion, Sirt2 plays different roles in various contexts, through the deacetylation of specific substrates.

Taken together, we have elucidated the molecular mechanism underlying the protective role of Sirt2 inactivation in APAP-induced liver injuries.

\section{MATERIALS AND METHODS}

See Supplementary Information for Materials and Methods.

\section{ACKNOWLEDGEMENTS}

We thank Dr. Chu-Xia Deng, Dr. S. G Rhee, and Dr. J. H. Ryu for providing the Sirt2 WT and Sirt2 KO mice. In addition, we thank S. H. Sung and S. Y. Oh for maintaining the Sirt2 WT and Sirt2 KO mice. This work was supported by the National Research Foundation of Korea (NRF-2017R1A2B4007400; S. H. Bae, NRF-2017R1D1A1B03032808; J. S. Park, NRF-2016R 1A5A1010764; 2015R1C1A1A01052558; Y. H. Lee) and a Faculty Research Grant from the Yonsei University College of Medicine (6-2014-0068; 6-2015-0099; S. H. Bae). It was also supported by a grant from the Korea Health Technology R\&D Project through the Korea Health Industry Development Institute (KHIDI), funded by the Ministry of Health \& Welfare, Republic of Korea (HI17C0913; HI16C0257; S. H. Bae, HI14C2476; Y. H. Lee). This research was also supported by the Korea Mouse Phenotyping Project (NRF-2016M3A9D 5A01952416) from the National Research Foundation, by the Bio \& Medical Technology Development Program of the NRF, funded by the Korean government (NRF-2017M3A9F3041234, NRF-2017R1A2B2009850) and the Brain Korea 21 PLUS Project for Medical Science, Yonsei University (to K. T. Nam).

\section{CONFLICTS OF INTEREST}

The authors have no conflicting interests.

\section{REFERENCES}

1. Ghabril M, Chalasani N and Bjornsson E (2010) Drug-induced liver injury: a clinical update. Curr Opin Gastroenterol 26, 222-226

2. James LP, Mayeux PR and Hinson JA (2003) Acetaminophen-induced hepatotoxicity. Drug Metab Dispos 31, 1499-1506

3. Ni HM, Williams JA, Yang H, Shi YH, Fan J and Ding WX (2012) Targeting autophagy for the treatment of liver diseases. Pharmacol Res 66, 463-474

4. Hinson JA, Roberts DW and James LP (2010) Mechanisms of acetaminophen-induced liver necrosis. Handb Exp Pharmacol, 369-405

5. Copple IM, Goldring CE, Jenkins RE et al (2008) The hepatotoxic metabolite of acetaminophen directly activates the Keap1-Nrf2 cell defense system. Hepatology 48, 1292-1301

6. Nagy G, Kardon T, Wunderlich L et al (2007) Acetaminophen induces ER dependent signaling in mouse liver. Arch Biochem Biophys 459, 273-279

7. Foufelle F and Fromenty B (2016) Role of endoplasmic reticulum stress in drug-induced toxicity. Pharmacol Res Perspect 4, e00211

8. Frye RA (2000) Phylogenetic classification of prokaryotic and eukaryotic Sir2-like proteins. Biochem Biophys Res 
Commun 273, 793-798

9. Morris BJ (2013) Seven sirtuins for seven deadly diseases of aging. Free Radic Biol Med 56, 133-171

10. Houtkooper RH, Pirinen E and Auwerx J (2012) Sirtuins as regulators of metabolism and healthspan. Nat Rev Mol Cell Biol 13, 225-238

11. Gomes P, Outeiro TF and Cavadas C (2015) Emerging Role of Sirtuin 2 in the Regulation of Mammalian Metabolism. Trends Pharmacol Sci 36, 756-768

12. Craig DG, Bates CM, Davidson JS, Martin KG, Hayes PC and Simpson KJ (2011) Overdose pattern and outcome in paracetamol-induced acute severe hepatotoxicity. $\mathrm{Br}$ J Clin Pharmacol 71, 273-282

13. Outeiro TF, Kontopoulos E, Altmann SM et al (2007) Sirtuin 2 inhibitors rescue alpha-synuclein-mediated toxicity in models of Parkinson's disease. Science 317, 516-519

14. Rumpf T, Schiedel M, Karaman B et al (2015) Selective Sirt2 inhibition by ligand-induced rearrangement of the active site. Nat Commun 6, 6263

15. Luthi-Carter R, Taylor DM Pallos J et al (2010) SIRT2 inhibition achieves neuroprotection by decreasing sterol biosynthesis. Proc Natl Acad Sci U S A 107, 7927-7932

16. Hu J, Ramshesh VK, McGill MR, Jaeschke $\mathrm{H}$ and Lemasters JJ (2016) Low Dose Acetaminophen Induces Reversible Mitochondrial Dysfunction Associated with Transient c-Jun N-Terminal Kinase Activation in Mouse Liver. Toxicol Sci 150, 204-215

17. Ni HM, Williams JA, Jaeschke H and Ding WX (2013) Zonated induction of autophagy and mitochondrial spheroids limits acetaminophen-induced necrosis in the liver. Redox Biol 1, 427-432

18. Lorz C, Justo P, Sanz A, Subira D, Egido J and Ortiz A (2004) Paracetamol-induced renal tubular injury: a role for ER stress. J Am Soc Nephrol 15, 380-389

19. Uzi D, Barda L, Scaiewicz V et al (2013) CHOP is a critical regulator of acetaminophen-induced hepatotoxicity. J Hepatol 59, 495-503

20. Kalinec GM, Thein P, Parsa A et al (2014) Acetaminophen and NAPQI are toxic to auditory cells via oxidative and endoplasmic reticulum stress-dependent pathways. Hear Res 313, 26-37

21. Bertolotti A, Zhang $Y$, Hendershot LM, Harding HP and Ron D (2000) Dynamic interaction of BiP and ER stress transducers in the unfolded-protein response. Nat Cell Biol 2, 326-332

22. Dong G, Liu $Y$, Zhang L, Huang S, Ding HF and Dong $Z$ (2015) mTOR contributes to ER stress and associated apoptosis in renal tubular cells. Am J Physiol Renal Physiol 308, F267-274

23. Wu M, Liu H, Fannin J et al (2010) Acetaminophen improves protein translational signaling in aged skeletal muscle. Rejuvenation Res 13, 571-579

24. Hong S, Zhao B, Lombard DB, Fingar DC and Inoki K (2014) Cross-talk between sirtuin and mammalian target of rapamycin complex 1 (mTORC1) signaling in the regulation of S6 kinase 1 (S6K1) phosphorylation. J Biol Chem 289, 13132-13141

25. Rack JG, VanLinden MR, Lutter T, Aasland R and Ziegler
$M$ (2014) Constitutive nuclear localization of an alternatively spliced sirtuin-2 isoform. J Mol Biol 426, 1677-1691

26. Mangas-Sanjuan V, Olah J, Gonzalez-Alvarez I et al (2015) Tubulin acetylation promoting potency and absorption efficacy of deacetylase inhibitors. $\mathrm{Br} J$ Pharmacol 172, 829-840

27. Chopra V, Quinti L, Kim J et al (2012) The sirtuin 2 inhibitor AK-7 is neuroprotective in Huntington's disease mouse models. Cell Rep 2, 1492-1497

28. Wu FL, Liu WY, Van Poucke $S$ et al (2016) Targeting endoplasmic reticulum stress in liver disease. Expert Rev Gastroenterol Hepatol 10, 1041-1052

29. Malhi H and Kaufman RJ (2011) Endoplasmic reticulum stress in liver disease. J Hepatol 54, 795-809

30. Hay $N$ and Sonenberg N (2004) Upstream and downstream of mTOR. Genes Dev 18, 1926-1945

31. Ni HM, Bockus $A$, Boggess $N$, Jaeschke $H$ and Ding WX (2012) Activation of autophagy protects against acetaminophen-induced hepatotoxicity. Hepatology 55, 222-232

32. Borude P, Bhushan B, Gunewardena S, Akakpo J, Jaeschke $\mathrm{H}$ and Apte $U$ (2018) Pleiotropic Role of p53 in Injury and Liver Regeneration after Acetaminophen Overdose. Am J Pathol 188, 1406-1418

33. Chen W, Zhang X, Fan J et al (2017) Tethering Interleukin-22 to Apolipoprotein A-I Ameliorates Mice from Acetaminophen-induced Liver Injury. Theranostics 7, 4135-4148

34. Wang J, Koh HW, Zhou L et al (2017) Sirtuin 2 aggravates postischemic liver injury by deacetylating mitogen-activated protein kinase phosphatase-1. Hepatology 65, 225-236

35. Arteaga $M$, Shang N, Ding $X$ et al (2016) Inhibition of SIRT2 suppresses hepatic fibrosis. Am J Physiol Gastrointest Liver Physiol 310, G1155-1168

36. Lu Z, Bourdi M, Li JH et al (2011) SIRT3-dependent deacetylation exacerbates acetaminophen hepatotoxicity. EMBO Rep 12, 840-846

37. Inoue T, Nakayama Y, Li Y et al (2014) SIRT2 knockdown increases basal autophagy and prevents postslippage death by abnormally prolonging the mitotic arrest that is induced by microtubule inhibitors. FEBS J 281, 26232637

38. Park SH, Zhu Y, Ozden O et al (2012) SIRT2 is a tumor suppressor that connects aging, acetylome, cell cycle signaling, and carcinogenesis. Transl Cancer Res 1, 15-21

39. Kim HS, Vassilopoulos A, Wang RH et al (2011) SIRT2 maintains genome integrity and suppresses tumorigenesis through regulating $\mathrm{APC} / \mathrm{C}$ activity. Cancer Cell 20, 487-499

40. Nie H, Chen $\mathrm{H}$, Han J et al (2011) Silencing of SIRT2 induces cell death and a decrease in the intracellular ATP level of PC12 cells. Int J Physiol Pathophysiol Pharmacol 3, 65-70

41. Hoffmann G, Breitenbucher F, Schuler $M$ and Ehrenhofer-Murray AE (2014) A novel sirtuin 2 (SIRT2) inhibitor with p53-dependent pro-apoptotic activity in non-small cell lung cancer. J Biol Chem 289, 5208-5216 